\title{
CATÁLOGO PRELIMINAR DE LAS ORCHIDACEAE DE LA ZONA PROTECTORA CERROS DE LA CARPINTERA, COSTA RICA
}

\author{
Carlos Ossenbach S. ${ }^{1,4}$, Mario Ossenbach S. ${ }^{2} \&$ Franco Pupulin ${ }^{3}$ \\ 'Fundación Charles H.Lankester, Sabanilla de Montes de Oca, Costa Rica \\ ${ }^{2}$ Asmocicu, Asociación Movimiento Cívico del Cantón de La Unión, Tres Ríos, Costa Rica \\ ${ }^{3}$ Jardín Botánico Lankester, Universidad de Costa Rica \\ ${ }^{4}$ Autor para correspondencia: caossenb@racsa.co.cr
}

Introducción. El gran botánico norteamericano Paul

C. Standley visitó nuestro país en dos ocasiones, en los veranos de 1923-24 y 1925-26, durante las cuales realizó el trabajo preliminar que conduciría, en 1937, a la publicación de su Flora of Costa Rica, primera flora de nuestro país en el siglo XX, si exceptuamos el trabajo inconcluso de Pittier en su Primitiae Florae Costaricensis.

Standley visitó La Carpintera en sus dos viajes a Costa Rica. En la introducción a dicha flora dice Standley (1937: 26): "Único entre los sitios de colecta de la región central es el Cerro de La Carpintera, cercano a Tres Ríos y Cartago, una montaña aislada y elevada que surge sobre los campos de Ochomogo [...]. Siento una atracción especial por La Carpintera, porque puede alcanzarse fácilmente a pie desde Cartago o Tres Ríos y por su flora, extraordinariamente variada, que nos ha ofrecido docenas de nuevas especies [...]. Las laderas altas de la montaña se han limpiado para potreros y hay senderos fáciles hasta el límite del bosque que cubre las partes altas, [...] alrededor de la cima. Se corta madera en los bordes del bosque, de manera que cada año el número de árboles se reduce. Sería un acto patriótico si este último resto de bella vegetación natural se conservara permanentemente como monumento nacional, de manera que las futuras generaciones pudieran ver lo bello que era su país en su estado primitivo.

El bosque alto de La Carpintera es denso y húmedo, y difícil de penetrar. En ninguna parte de Costa Rica he visto una vegetación tan variada y exuberante. Hay profusión de árboles raros, muchos helechos arborescentes, un crecimiento exuberante de innumerables tipos de epífitas [...] numerosos helechos y gran variedad de bellas flores...
Lo más memorable de un ascenso a La Carpintera es el hecho de que todo el día se entretiene uno con los aullidos de unos cuantos "nausingos" que de alguna manera han sobrevivido en su restringido y aislado hábitat. En ningún otro lugar tan cercano a las ciudades de Costa Rica es posible oír las magníficas voces de estos grandes, negros, monos aulladores, los más grandes simios de Centroamérica."

Como veremos luego, el deseo de Standley de proteger La Carpintera no empezaría a tomar forma hasta en 1976.

\section{Antecedentes.}

a) Datos generales. La Zona Protectora Cerros de La Carpintera fue creada mediante Decreto Ejecutivo 6112-A del 23 de junio de 1976. La zona comprende una superficie de aproximadamente 2396 hectáreas y su manejo se rige por lo que determina la Ley General Forestal No. 7575 del 13 de febrero de 1996. Cubre la parte alta de los Cerros de La Carpintera y se reparte entre cuatro cantones (La Unión, Cartago, Desamparados y Curridabat), aunque la mayor porción, la mejor conservada y la de mayor belleza escénica es la que se encuentra en el Cantón de La Unión. Para los habitantes de Tres Ríos, los Cerros de La Carpintera son el símbolo del Cantón y es un orgullo para muchos de ellos conocer al dedillo los senderos, las grutas, los bosques y las fincas de La Carpintera. Igualmente, es un sentir generalizado en el Cantón que La Carpintera está seriamente amenazada y que es necesario poner en práctica medidas que garanticen su conservación.

b) Importancia biológica. En los Cerros de La Carpintera se encuentra una parte de los últimos bosques primarios de altura remanentes del Valle 
Central de Costa Rica. La Carpintera es la localidad tipo (locus typicus o clasicus) de varias especies de plantas. Su importancia específica deriva de su posición entre la Cordillera Volcánica Central y la Cordillera de Talamanca, lo que convierte a estos cerros en un corredor biológico de suma importancia. Dicho corredor está cada vez más amenazado, ya que la autopista Curridabat-Cartago y la urbanización de ambos lados de dicha pista constituyen un corte cada vez más profundo en este corredor. El área tan reducida de bosque primario (aproximadamente 618 hectáreas) hace pensar que es difícil que este ecosistema pueda ser viable a largo plazo, por lo que es necesario no solamente garantizar su protección, sino que también es indispensable fortalecer su conexión con las dos cordilleras a las que sirve de puente. Censos de aves realizados en los años 2000 y 2001 por la Asociación Ornitológica de Costa Rica con el apoyo de miembros de la Asociación Movimiento Cívico del Cantón de La Unión han permitido identificar 164 especies diferentes. Aunque no tenemos conocimiento de estudios exhaustivos sobre flora y fauna de esta zona, es de presumir que la biodiversidad sea similar a la detectada en el campo de las aves.

c) Importancia como fuente de recursos hídricos. Como fuente de recursos hídricos, La Carpintera juega también un papel importantísimo para ambas vertientes, la Atlántica y la Pacífica. Poblaciones en ambos lados de los Cerros de La Carpintera dependen de los manantiales que nacen allí para su abastecimiento de agua potable.

d) Descripción. La Zona Protectora Cerros de La Carpintera está localizada al sureste de San José, a unos cinco a siete kilómetros del centro de la ciudad. La cima de los cerros es divisoria de aguas entre la vertiente Atlántica y la vertiente Pacífica. Los tres picos más altos (los que se conocen propiamente como La Carpintera) tienen alturas entre 1800 y 1855 m. Otras alturas importantes son el Cerro Quirazú (1794 m), Alto Lima (1651 m) y Alto Negro (1747 m). Las 2396 hectáreas de la Zona Protectora se distribuyen entre los cantones de La Unión (974 has), Cartago (964 has), Desamparados (429 has) y Curridabat (28 has). El clima en los Cerros de La Carpintera presenta el patrón típico del Valle Central, con una estación seca de diciembre a abril y otra lluviosa de mayo a noviembre. Los datos anuales promedio varían de acuerdo a la altura y la ubicación. Son:

$\begin{array}{ll}\text { Precipitación: } & \text { entre } 1500 \text { y } 2500 \mathrm{~mm} \\ \text { Temperatura: } & \text { de } 15 \text { a } 23^{\circ} \mathrm{C} \\ \text { Humedad relativa: } & \text { entre } 70 \text { y } 90 \%\end{array}$

Los Cerros de La Carpintera forman parte del grupo de intrusiones que caracterizan la zona sur de San José, tanto en el flanco oriental como en el occidental. Las principales formaciones estructurales son: Formación Térraba, Formación San Miguel y Formación Coris. Por tanto, encontramos en estos cerros areniscas, lutita negra, calizas, cuarzo y arenas silíceas, entre otros.

e) Tenencia y uso de la tierra. La tierra en la Zona Protectora de La Carpintera se encuentra bajo régimen de propiedad privada. Al 23 de noviembre de 1999 la Oficina de Atención al Usuario del Ministerio del Ambiente y la Energía (MINAE) tenía registradas 928 propiedades desde $100 \mathrm{~m}^{2}$ hasta fincas de 450 has. Según el Sistema Nacional de Áreas de Conservación (SINAC), el uso de la tierra en la Zona Protectora se distribuye así:

\begin{tabular}{lcc}
\hline Bosque primario & 618 has & $26 \%$ \\
Bosque secundario & 194 has & $8 \%$ \\
Charral & 57 has & $2 \%$ \\
Cultivos y pasto & 769 has & $32 \%$ \\
Suelo desnudo y ciudades & 256 has & $11 \%$ \\
Reforestación/recuperación & 502 has & $21 \%$ \\
\hline
\end{tabular}

Estos datos no concuerdan exactamente con estudios de años anteriores e indican tanto diferencias en metodología como una evolución en el uso de la tierra, principalmente en lo que se refiere a urbanización.

\section{El proyecto.}

a) Objetivos. Al igual que los censos de aves realizados por la Asociación Ornitológica de Costa Rica en conjunto con el Movimiento Cívico del Cantón de La Unión, la realización de un inventario y la publicación de un catálogo de orquídeas en la Zona Protectora Cerros de La Carpintera tiene como fin destacar la importancia ecológica de dicha zona, ayudando a fundamentar la necesidad de su preservación. 
b) Metodología. 1. En una primera etapa se analizó la información bibliográfica existente, que en muchos casos contiene datos de colecciones botánicas de Orchidaceae realizadas en La Carpintera. 2. El siguiente paso consistió en analizar los datos de colecta de los ejemplares de orquídeas conservados en los principales herbarios nacionales. 3. Por último, se realizaron giras al campo para corroborar y ampliar la información obtenida en las etapas previas.

\section{Las colecciones históricas.}

a) Generalidades. No conocemos ninguna colecta de Orchidaceae realizada en La Carpintera por los primeros exploradores botánicos que recorrieron Costa Rica durante el siglo XIX. En la obra clásica de Reichenbach sobre las orquídeas de Centroamérica, Beiträge zu einer Orchideenkunde Central-America's ("Aportes a una orquideología de la América Central”, Reichenbach 1866), La Carpintera no figura como lugar de origen para ninguna de las especies ahí descritas, recolectadas por Oersted, von Warscewicz y Wendland. Es por ello que la aproximación histórica a este Catálogo Preliminar de las Orchidaceae de La Carpintera debe iniciarse con el estudio de la gran obra de Rudolf Schlechter: Beiträge zur Orchideenkunde von Zentralamerika ("Aportes a una orquideología de Centroamérica”, Schlechter 1923) y a las obras fundamentales de Oakes Ames Schedulae Orchidianae (1922-1930) y su capítulo de Orchidaceae para la Flora of Costa Rica de Standley (Ames 1937).

b) Las orquídeas de La Carpintera en la obra de Rudolf Schlechter. Rudolf Schlechter (1872-1925) fue sin duda el más prominente orquideólogo de la primera mitad del siglo XX. Apasionado de su trabajo, se dice del que fuera director del herbario del Jardín Botánico de Berlín que pretendía publicar una nueva especie de orquídea durante cada día de su vida. Los ejemplares encontrados por los más connotados colectores locales de las primeras décadas del siglo XX fueron enviados a Schlechter para su identificación. Es en las obras de Schlechter donde aparece por vez primera el nombre de La Carpintera en la bibliografía orquideológica de nuestro país, al lado del nombre de los hermanos Curt y
Alfred Brade. Fueron los Brade, jardineros alemanes establecidos en Costa Rica, aparentemente los únicos corresponsales de Schlechter que colectaron en La Carpintera, con la excepción de la colecta de Pittier y Tonduz que dio base a la descripción de Malaxis carpinterae (Fig. 1). De las colecciones realizadas por los hermanos Brade provenientes de La Carpintera, Schlechter enumera, en su obra ya citada, un total de 15 especies en 10 géneros diferentes.

Entre ellas, como nuevas para la ciencia, fueron descritas por primera vez 4 especies:

\section{Lepanthes blephariglossa \\ Lepanthes ciliisepala \\ Pleurothallis homalantha}

Pleurothallis $(=$ Stelis) carpinterae

c) Las orquideas de La Carpintera en la obra de Oakes Ames. Oakes Ames (1874-1950), hijo de familia culta y acaudalada de Nueva Inglaterra, se graduó en la Universidad de Harvard en 1899 y fue nombrado poco tiempo después como director del Jardín Botánico de la Universidad. A partir del tercer fascículo de sus Schedulae Orchidianae encontramos con cada vez mayor frecuencia especies nuevas descritas con base en colecciones realizadas por Tonduz, Standley y Lankester. Entre ellas encontramos 8 especies en 5 géneros colectadas en La Carpintera (en adición a las ya mencionadas anteriormente por Schlechter).

Como nuevas para la ciencia, Ames describe 4 especies:

\section{Coccineorchis standleyi \\ Lepanthes fimbriata \\ Pleurothallis (= Specklinia) strumosa \\ Pleurothallis (= Stelis) pompalis}

d) Las orquideas de La Carpintera en la Flora of Costa Rica de Paul C. Standley. En su tratado de Orchidaceae para la Flora of Costa Rica, Ames (1937) resume los conocimientos que existían hasta aquel entonces sobre las orquídeas de Costa Rica. Como colectadas en La Carpintera se enumeran aquí 16 especies en 11 géneros, sin contar las especies que ya figuraban en las obras anteriores de Schlechter y del mismo Ames. 


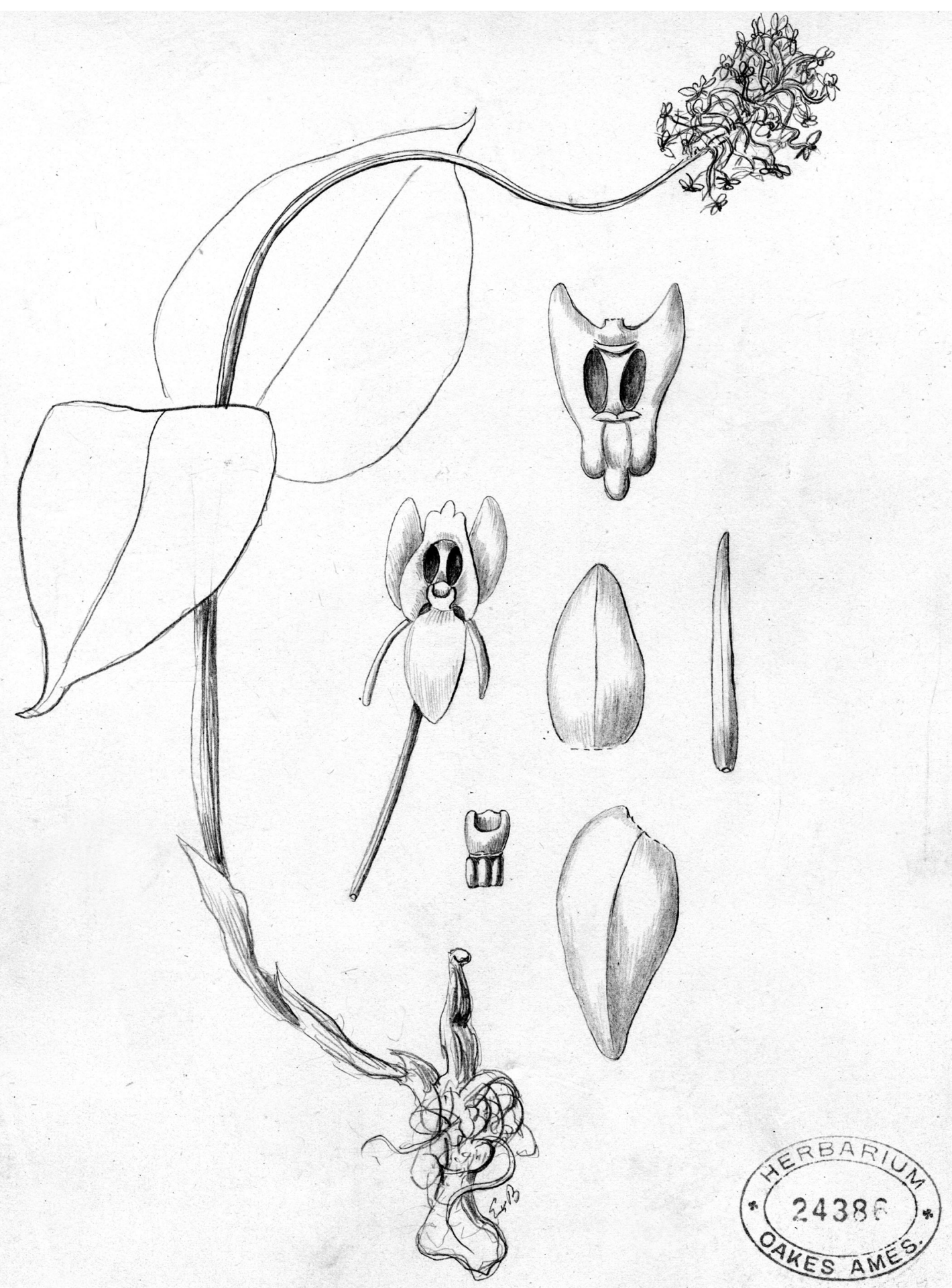

Fig. 1. Dibujo de Malaxis (Microstylis) carpinterae (Schltr.) Ames, realizado bajo la supervisión de Schlechter y conservado en el Herbario de Oakes Ames, Universidad de Harvard. Al pie del dibujo hay una nota que dice: "Costa Rica, forets de La Carpintera, VIII, 1891 - H. Pittier \& Tonduz". 


\section{Orquídeas de la Carpintera en los herbarios nacionales.}

a) Generalidades. Las bases de datos informatizadas nos permiten hoy día un rápido acceso a la información contenida en herbarios. De los tres principales herbarios nacionales, el del Instituto Nacional de Biodiversidad (INBio) es el único que no cuenta con ejemplares de Orchidaceae colectados en La Carpintera.

b) Orquídeas de La Carpintera en el Herbario Nacional. El Herbario Nacional cuenta con un total de 13 colecciones de orquídeas realizadas en $\mathrm{La}$ Carpintera, en 9 géneros diferentes. Interesantes desde el punto de vista histórico son dos colectas de Louis O. Williams, del año 1949.

c) Orquídeas de La Carpintera en el Herbario de la Universidad de Costa Rica. El Herbario USJ es el más rico en especímenes procedentes de La Carpintera, con 25 especies en 15 géneros, la mayoría recolectados por Carlos O. Morales en años recientes.

d) Orquídeas de La Carpintera en la colección viva del Jardín Botánico Lankester. En la colección viva del Jardín Botánico Lankester se encuentran 4 especies y un género adicionales, provenientes de colectas en los Cerros de La Carpintera. Se encuentran además en el Jardín ejemplares sin identificar de los géneros Maxillaria, Erythrodes y Govenia.

\section{Catálogo de las Orchidaceae de los Cerros de La} Carpintera. La lista preliminar de Orchidaceae recolectadas en La Carpintera que se agrega a continuación (Tabla 1) incluye únicamente los géneros y especies enumerados por Schlechter y Ames en la bibliografía citada, y las especies que se encuentran depositadas en los herbarios nacionales.

Esta primera lista está integrada por 67 especies en 34 géneros diferentes (68 especies en 35 géneros si incluyéramos las especies no identificadas en el Jardín Botánico Lankester). Es probable que las giras al campo que se realizarán para complementar y corroborar la información bibliográfica y la contenida en los herbarios nacionales arroje un número de especies mucho mayor.

De las contenidas en la lista, un total de 10 especies fueron descritas por primera vez con base en colectas realizadas en La Carpintera.

De las 67 especies mencionadas, 10 son endémicas de Costa Rica, de las cuales una (Malaxis carpinterae) es probablemente endémica de La Carpintera (Fig. 1).

\section{AgRAdECIEMIENTOS}

Los autores agradecen a Armando Estrada y Emily Serrano por la información proporcionada acerca de las colecciones del Museo Nacional de Costa Rica (CR), y a Carlos O. Morales por la ayuda brindada en el Herbario de la Universidad de Costa Rica (USJ) y por sus observaciones de campo.

\section{Literatura CitADA}

Ames, O. 1992-1930. Schedulae Orchidianae, 1-10. Boston. Reimpresión por Bishen Singh Mahendra Pal Singh, India, 1983.

Ames, O. 1937. Orchidaceae. In P.C. Standley, Flora of Costa Rica. Part 1. Field Museum of Natural History, Chicago, Botanical Series.

Instituto Nacional de Biodiversidad. 2003. Página "web" http://www.inbio.ac.cr

Meza Ocampo, T. 1979. Consideraciones generales sobre la morfoestructura y el modelado climático en los Cerros de La Carpintera y su relación con el conjunto Irazú, Costa Rica. Tesis, Universidad de Costa Rica.

Ossenbach, C. en prensa. Breve historia de la orquideología de Costa Rica. San José, Editorial de la Universidad de Costa Rica.

Pupulin, F. 2002. Catálogo revisado y anotado de las Orchidaceae de Costa Rica. Lankesteriana 4: 1-88.

Reichenbach, H.G. 1866. Beiträge zu einer Orchideenkunde Central-America's. Hamburgo.

Schlechter. R. 1917. Beiträge zur Orchideenkunde von Zentralamerika. I. Orchidaceae Powellianae Panamenses. Repert. Sp. Nov. Regni Veg. 17. Verlag des Repertoriums, Dahlem bei Berlin. Reimpresión por Otto Koeltz Antiquariat, Königstein, R.F.A., 1980.

Schlechter. R. 1923. Beiträge zur Orchideenkunde von Zentralamerika. II. Additamenta ad Orchideologiam Costaricensis. Repert. Sp. Nov. Regni Veg. 19. Verlag des Repertoriums, Dahlem bei Berlin. Reimpresión por Otto Koeltz Antiquariat, Königstein,, 1980.

Standley, P.C. 1937. Flora of Costa Rica. Part 1. Field Museum of Natural History, Chicago, Botanical Series.

Torres Guerrero, A. 2002. Programa ambiental para la consolidación de la Zona Protectora Cerros de La Carpintera. Proyecto de Ley, Asamblea Legislativa, República de Costa Rica, marzo 2002. 
Tabla 1. Catálogo de las Orchidaceae de los Cerros de La Carpintera. El asterisco (*) indica las especies endémicas de Costa Rica.

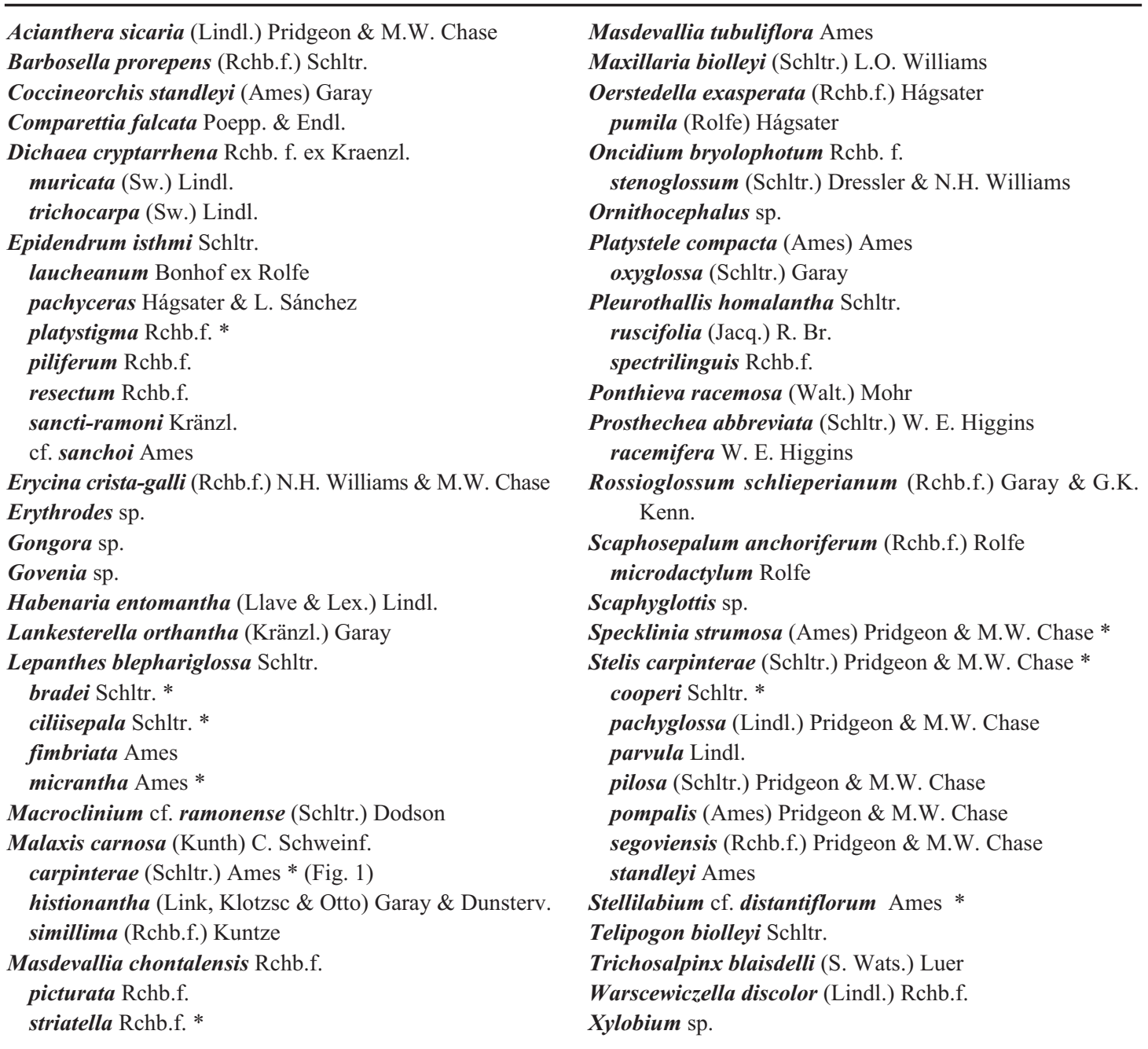

Carlos Ossenbach es cofundador de la Fundación Charles H. Lankester y sus dos intereses principales son la historia de la orquideología y la bibliografía sobre orquídeas.

Mario Ossenbach es Presidente de la Asociación Ornitológica de Costa Rica y Tesorero de la Asociación Movimiento Cívico de Tres Ríos. Se ha dedicado con gran interés a la conservación de las áreas protegidas de Costa Rica y sobre todo de los Cerros de La Carpintera.

Franco Pupulin es profesor de la UCR y trabaja como investigador en el Jardín Botánico Lankester. Realiza estudios de sistemática y evolución de las orquídeas, y trabajos florísticos en áreas protegidas. 